\title{
Impact of High Density Planting and Weed Management Practices on Growth Parameters and Phenology of Bt Cotton
}

\author{
B. Madavi ${ }^{1}$, P. Leela Rani ${ }^{2 *}$, G. Sreenivas ${ }^{3}$ and K. Surekha ${ }^{4}$ \\ ${ }^{1}$ Department of Agronomy College of Agriculture, Rajendranagar, Hyderabad, PJTSAU, India \\ ${ }^{2}$ AICRP on Weed Management, Rajendranagar, Hyderabad, PJTSAU, India \\ ${ }^{3}$ Agro Climate Research Centre, ARI, Rajendranagar, Hyderabad PJTSAU, India \\ ${ }^{4}$ Indian Institute of Rice Research, Rajendranagar, Hyderabad, IIOR, India \\ *Corresponding author
}

\begin{tabular}{|c|c|}
\hline \multicolumn{2}{|r|}{ A B S T R A C T } \\
\hline & \multirow{6}{*}{ 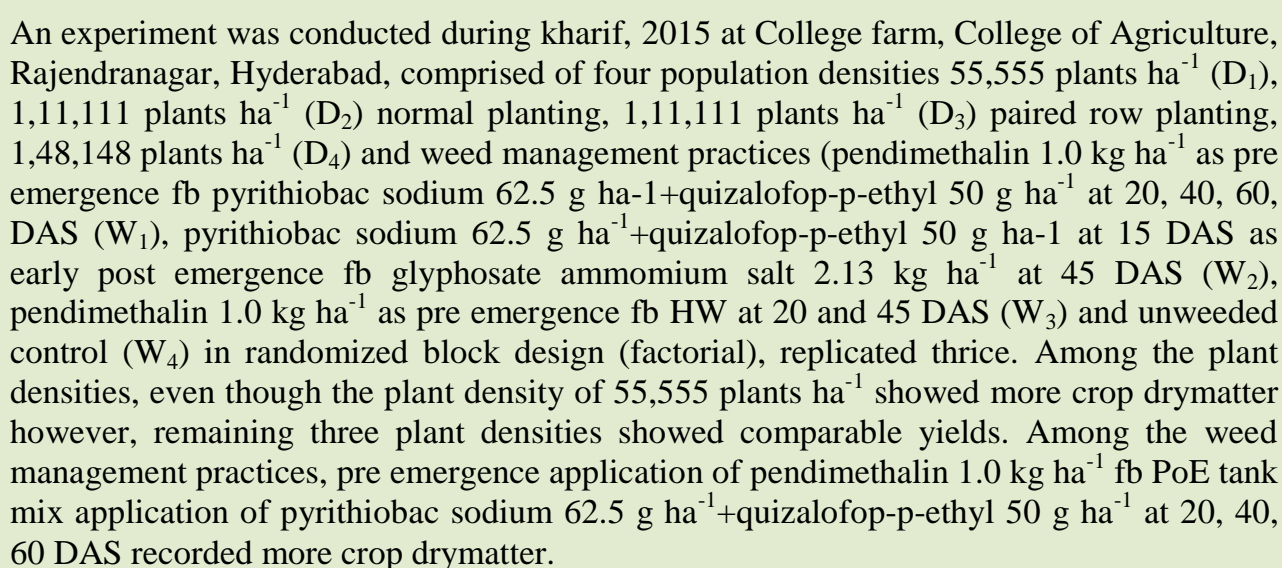 } \\
\hline Keywords & \\
\hline $\begin{array}{l}\text { High density, } \\
\text { Planting, Growth, } \\
\text { cotton. }\end{array}$ & \\
\hline Article Info & \\
\hline $\begin{array}{l}\text { Accepted: } \\
\text { 16 August } 2017 \\
\text { Available Online: } \\
\text { 10 September } 2017\end{array}$ & \\
\hline & \\
\hline
\end{tabular}

\section{Introduction}

India is the second largest producer of cotton in the world after China accounting for about 25.73 per cent of the world cotton production. In India, area under cotton is 105 lakh ha with production of 351 lakh bales and $568 \mathrm{~kg} \mathrm{ha}^{-1}$ productivity Anonymous (2016-17).

In India, Maharashtra stands first in area of cotton followed by Gujarat and Telangana. Area in Telangana under cotton is 12.50 lakh ha with production of 48 lakh bales and productivity $653 \mathrm{~kg} \mathrm{ha}^{-1}$ Anonymous (201617).
Sustain the cotton productivity in rainfed soils need to practice high density planting systems, with narrow and ultra-narrow spacing. Weed competition is severe during its initial growth stage. Venugopalan et al., (2009) reported a reduction in yield due to weeds in cotton crop to the extent of 50 to 85 per cent. Jain (1982) reported that, weeds removed as high as $48-50 \mathrm{~kg} \mathrm{~N}, 8-15 \mathrm{~kg} \mathrm{P}$ and $48-50 \mathrm{~kg} \mathrm{~K} \mathrm{ha}^{-1}$. Thus, if proper weed control measures are followed, there would be greater availability of nutrients and moisture for the benefit of crop (Jalis and Shah, 1982). 
In cotton First 60 DAS was the most critical period for crop-weed competition, mostly in kharif season due to incessant rains, hand weeding and inter-cultivation become difficult in cotton. Farmers were forced to use herbicides for weed control in cotton. Hence, there is a need for selection of herbicides to control emerging weeds during the crop growth period. So to attain a season long weed control, integration of chemical, mechanical and cultural methods holds a great promise in crop production. Hence, integrated weed management in cotton play important role in increasing crop production. Pre emergence herbicides at recommended doses are generally capable of controlling annual weeds upto a period of 30 days (Pawar et al., 2000). Braret al., (1995) stated that pre emergence application of pendimethalin @ $1.5 \mathrm{~kg} \mathrm{ha}^{-1}$ followed by one hoeing at 30 DAS was effective for the control of annual broad leaved and grassy weeds like Trianthema portulacastrum and Eleusine indica. The concentration of PRE emergence herbicide was decreased beyond 30 days after application, so which effect the increases the weed population. Due to regular monsoon rains farmers were unable to intercultivate which leads to increase the weeds population and compete with crop plants and finally reduce the seed cotton yield. Hence, there is a need to go for sequential application of PRE followed by POE herbicides to manage the late emerging weeds to eliminate weed competition throughout the critical period (Pawar et al., 2000).

\section{Materials and Methods}

A field experiment was conducted during kharif, 2015 at College farm, College of Agriculture, Professor Jayashankar Telangana State Agricultural University, Rajendranagar, Hyderabad. The farm is geographically situated an altitude of $542.6 \mathrm{~m}$ above mean sea level on 18 ' $50 \mathrm{o} \mathrm{N}$ latitude and $77.53 \mathrm{o} \mathrm{E}$ longitude). The soil of the experimental field was sandy loam in texture, low in available $\mathrm{N}$ $\left(250 \mathrm{~kg} \mathrm{ha}^{-1}\right)$, medium in phosphorus $(21.68$ $\left.\mathrm{kg} \mathrm{P}_{2} \mathrm{O}_{5} \mathrm{ha}^{-1}\right)$ and high in potassium $(685.6 \mathrm{~kg}$ $\mathrm{K}_{2} \mathrm{O}$ ha $^{-1}$ ).

The experiment was conducted to test the impact of four planting densities 55,555 plants $\mathrm{ha}^{-1}(60 \mathrm{~cm} \times 30 \mathrm{~cm}), 1,11,111$ plants $\mathrm{ha}^{-1}(60 \mathrm{~cm} \times 15 \mathrm{~cm})$ normal planting, $1,11,111$ plants $\mathrm{ha}^{-1}(60 \mathrm{~cm} \times 15 \mathrm{~cm})$ paired row planting, 1,48,148 plants $\mathrm{ha}^{-1}(45 \mathrm{~cm} \times 15$ $\mathrm{cm})$ and weed management practices viz., pendimethalin $1.0 \mathrm{~kg} \mathrm{ha}^{-1}$ as pre emergence $\mathrm{fb}$ pyrithiobac sodium $62.5 \mathrm{~g} \mathrm{ha}^{-1}+$ quizalofop-pethyl $50 \mathrm{~g} \mathrm{ha}^{-1}$ at 20, 40, 60, DAS (W $)$, pyrithiobac sodium $62.5 \mathrm{~g} \mathrm{ha}^{-1}+$ quizalofop-pethyl $50 \mathrm{~g} \mathrm{ha}^{-1}$ at 15 DAS as early post emergence $\mathrm{fb}$ glyphosate ammomium salt $2.13 \mathrm{~kg} \mathrm{ha}^{-1}$ at $45 \mathrm{DAS}\left(\mathrm{W}_{2}\right)$, pendimethalin $1.0 \mathrm{~kg} \mathrm{ha}^{-1}$ as pre emergence fb HW at 20 and 45 DAS $\left(\mathrm{W}_{3}\right)$ and unweeded control $\left(\mathrm{W}_{4}\right)$ on yield and quality of $\mathrm{Bt}$ cotton in randomized block design (factorial), replicated thrice. The crop was sown on 26th june. Other cultural operations and plant protections measures were followed as per the recommendations. During the crop period rainfall of $404.3 \mathrm{~mm}$ was received in 28 rainy days in 2015 as against the decennial average of $683.1 \mathrm{~mm}$ received in 35 rainy days for the corresponding period. Five plants were randomly selected in each plot and tagged to record the regular observations. Four pickings of seed cotton yield was taken from each treatment for recording final yield data.

\section{Results and Discussion}

\section{Plant height (cm)}

\section{Effect of plant densities}

Weed with 55,555 plants $\mathrm{ha}^{-1}\left(\mathrm{D}_{1}\right)$ at all the growth stages except at 60 DAS, but at 30 , 120 DAS and at final harvest stage did not differ significantly with other densities (Tables 1). However, at 60 DAS more plant 
height was observed with $1,11,111$ plants $^{-1}$ $\left(\mathrm{D}_{2}\right)$ normal planting and was significantly superior over $1,11,111$ plants ha ${ }^{-1}\left(D_{3}\right)$ paired row and $1,48,148$ plants ha ${ }^{-1}\left(D_{4}\right)$, but it was on par with 55,555 plants ha ${ }^{-1}\left(D_{1}\right)$. At 90 DAS more plant height was recorded with 55,555 Linear increase in plant height was observed up to the end of the crop growing season. Increased plant height was notice plants $\mathrm{ha}^{-1}\left(\mathrm{D}_{1}\right)$ and on par plant height was observed with $1,48,148$ plants $\mathrm{ha}^{-1}\left(\mathrm{D}_{4}\right)$, in turn this was on par with $1,11,111$ plants ha ${ }^{-1}$ $\left(D_{3}\right)$ paired row, which in turn on par with $1,11,111$ plants $\mathrm{ha}^{-1}\left(\mathrm{D}_{2}\right)$ normal planting. Similar trend was observed at 120 DAS and final harvest stage even though they did not differ significantly.

The taller plants at higher plant density late in the season might be due to inter plant competition for nutrients and light. Further the availability of horizontal space for individual plant at closer spacing reduced, due to which intense inter plant competition for nutrient and light suppressed node appearance and plants grew taller in respect of vertical space (Wang et al., 2011).

\section{Effect of weed management practices}

Weed management practices showed significant influence on plant height at all the stages. Increase in plant height was observed with early PoE tank mix application of pyrithiobac sodium $62.5 \mathrm{~g} \mathrm{ha}^{-1}+$ quizalofop-pethyl $50 \mathrm{~g} \mathrm{ha}^{-1}$ at 15 DAS fb directed spray of glyphosate ammonium salt $2.13 \mathrm{~kg} \mathrm{ha}^{-1}$ at 45 DAS $\left(\mathrm{W}_{2}\right)$ at all the stages except at $30 \mathrm{DAS}$, where pre emergence application of pendimethalin $1.0 \mathrm{~kg} \mathrm{ha}^{-1} \mathrm{fb}$ PoE tank mix application of pyrithiobac sodium $62.5 \mathrm{~g}$ ha ${ }^{1}$ +quizalofop-p-ethyl $50 \mathrm{~g} \mathrm{ha}^{-1}$ at 20, 40, 60 DAS $\left(\mathrm{W}_{1}\right)$ recorded more plant height and was significantly superior over all other weed management practices, in turn these were on par with each other. But, at 60 and 120 DAS significantly more plant height was noticed with $\mathrm{W}_{2}$ and was superior over all other weed management practices, this was followed by pre emergence application of pendimethalin $1.0 \mathrm{~kg} \mathrm{ha}^{-1} \mathrm{fb}$ PoE tank mix application of pyrithiobac sodium $62.5 \mathrm{~g} \mathrm{ha}^{-1}+$ quizalofop-pethyl $50 \mathrm{~g} \mathrm{ha}^{-1}$ at 20, 40, 60 DAS $\left(\mathrm{W}_{1}\right)$ and pendimethalin $1.0 \mathrm{~kg} \mathrm{ha}^{-1}$ (PE) fb $\mathrm{HW}$ at 20 and 45 DAS $\left(\mathrm{W}_{3}\right)$ treatments, in turn these showed on par plant height with each other.

However, at 90 DAS and at final harvest stage, early PoE application of pyrithiobac sodium $62.5 \mathrm{~g} \mathrm{ha}^{-1}+$ quizalofop-p-ethyl $50 \mathrm{~g}$ $\mathrm{ha}^{-1}$ at 15 DAS fb directed spray of glyphosate ammonium salt $2.13 \mathrm{~kg} \mathrm{ha}^{-1}$ at $45 \mathrm{DAS}\left(\mathrm{W}_{2}\right)$ treatment did not differ with $\mathrm{W}_{1}$ and $\mathrm{W}_{3}$ treatments. At all the stages significantly the lowest plant height was recorded with unweeded control $\left(\mathrm{W}_{4}\right)$ treatment. The decreased plant height was might be due to more weed dry matter and weed density at all the growth stages. The increased plant height in $\mathrm{W}_{2}$ due to reduced weed dry matter and weed density with reduced broad and narrow leaf weeds from 60 DAS might be due to directed spray of non-selective glyphosate ammonium salt at 45 DAS. Similar results were reported by Ali et al., (2013).

\section{Interaction effect}

Plant densities and weed management practices did not show any significant influence on plant height at any crop growth stage of the Bt cotton.

\section{Crop drymatter (g plant $\left.{ }^{-1}\right)$}

\section{Effect of plant densities}

Plant densities and weed management practices did not exert any significant influence on crop drymatter at 30 DAS (Table 2). Significant increase in CDM was observed with plant density of 55,555 plants ha ${ }^{-1}\left(D_{1}\right)$ 
and was on par with $1,11,111$ plants $\mathrm{ha}^{-1}\left(\mathrm{D}_{2}\right)$ normal planting, in turn this was on par with $1,11,111$ plants $\mathrm{ha}^{-1}\left(\mathrm{D}_{3}\right)$ paired row planting, at all the growth stages, again these were was on par with $1,48,148$ plants $\mathrm{ha}^{-1}\left(\mathrm{D}_{4}\right)$ at 60 DAS and at final harvest. However, at 90 DAS and 120 DAS $1,11,111$ plants ha ${ }^{-1}\left(D_{3}\right)$ paired row planting poduced significantly more drymatter over $1,48,148$ plants $\mathrm{ha}^{-1}$ (D4).

Drymatter plant ${ }^{-1}$ was higher with wider spacing, this might be due to more canopy development under wider spacing (Devraj et al., 2011). The marked improvements in growth and yield attributing character was brought due to the more availability of solar radiation and that helps to synthesis and partitioning of assimilates to individual plant under wider spacing, which ultimately translocates assimilates from source to sink that leads to significant increment in growth attributes in respect of weight and diameter of plant (Bhalerao et al., 2010 and Dhillon et al., 2006).

\section{Effect of weed management practices}

Increase in crop drymatter was observed with pre emergence application of pendimethalin $1.0 \mathrm{~kg} \mathrm{ha}^{-1} \mathrm{fb}$ PoE tank mix application of pyrithiobac sodium $62.5 \mathrm{~g} \mathrm{ha}^{-1}+$ quizalofop-pethyl $50 \mathrm{~g} \mathrm{ha}^{-1}$ at 20, 40,60 DAS $\left(\mathrm{W}_{1}\right)$ at all the crop growth stages and was not differed significantly with pre emergence application of pendimethalin $1.0 \mathrm{~kg} \mathrm{ha}^{-1}$ (PE) fb HW at 20 and 45 DAS $\left(\mathrm{W}_{3}\right)$ and were significantly superior over rest of the weed management practices except at 60 DAS, where $\mathrm{W}_{3}$ treatment was on par with early $\mathrm{PoE}$ of pyrithiobac sodium $62.5 \mathrm{~g} \mathrm{ha}^{-1}+$ quizalofop-pethyl $50 \mathrm{~g} \mathrm{ha}^{-1}$ at 15 DAS fb directed spray of glyphosate ammonium salt $2.13 \mathrm{~kg} \mathrm{ha}^{-1}$ at 45 DAS $\left(\mathrm{W}_{2}\right)$ at remaining stages. The remaining two treatments differed significantly with each other and the lowest crop drymatter was recorded with unweeded control $\left(\mathrm{W}_{4}\right)$ treatment at all the crop growth stages.

The observed more crop drymatter production of cotton in $\mathrm{W}_{1}, \mathrm{~W}_{3}$ and $\mathrm{W}_{2}$ treatments was due to higher accumulation of photosynthates in leaves, stem and reproductive parts due to increase in the uptake of nutrients (Hiremath et al., 2013), thereby increase in the growth of components and it lead to the higher dry matter production plant ${ }^{-1}$ due to less weed dry matter and density while it was lower in unweeded control treatment $\left(\mathrm{W}_{4}\right)$.

\section{Interaction effect}

Plant densities and weed management practices did not show any significant influence on crop dry matter of Bt cotton at all the crop growth stages.

Main stem nodes (no. plant $^{-1}$ )

\section{Effect of plant densities}

The node number plant $^{-1}$ increased progressively up to the end of the crop growing season (Tables 3). Node number differed significantly during early stage of the crop growth only (30 DAS, 60 DAS and 90 DAS) and did not show any significant influence at later stage of crop growth (120 DAS and at final harvest).

More number of main stem nodes plant ${ }^{-1}$ was noticed with plant density of 55,555 plants ha ${ }^{1}\left(D_{1}\right)$ at all the crop growth stages and was on par with $1,11,111$ plants $\mathrm{ha}^{-1}\left(\mathrm{D}_{2}\right)$ normal planting, in turn it was on par with $1,11,111$ plants $\mathrm{ha}^{-1}\left(\mathrm{D}_{3}\right)$ paired row planting, again this was in turn on par with $1,48,148$ plants ha $^{-1}$ $\left(\mathrm{D}_{4}\right)$ at 30 and 60 DAS. However, at 90 DAS plant density of 55,555 plants ha ${ }^{-1}\left(\mathrm{D}_{1}\right)$ was on par with $1,11,111$ plants ha ${ }^{-1}\left(\mathrm{D}_{3}\right)$ paired row planting and 1,48,148 plants ha ${ }^{-1}\left(\mathrm{D}_{4}\right)$. Similar trend was observed with 120 DAS and at final harvest even though they were not differed significantly. 
Total numbers of nodes were higher for low plant density than higher density as the nodes per plant were inversely related to the plant densities (Seibert, 2006 and Stewart et al., 2006). But from the present study at 90 DAS increase in plant height was observed as the plant density was increased from $1,11,111$ plants ha ${ }^{-1}$ to $1,48,148$ plants ha ${ }^{-1}$ due to inter plant competition within the row.

\section{Effect of weed management practices}

Significant increase in main stem node number was observed with early PoE tank mix application of pyrithiobac sodium $62.5 \mathrm{~g}$ $\mathrm{ha}^{-1}$ quizalofop-p-ethyl $50 \mathrm{~g} \mathrm{ha}^{-1}$ at 15 DAS fb by directed spray of glyphosate ammonium salt $2.13 \mathrm{~kg} \mathrm{ha}^{-1}$ at $45 \mathrm{DAS}\left(\mathrm{W}_{2}\right)$ except at 30 DAS and was on par with pre emergence application of pendimethalin $1.0 \mathrm{~kg} \mathrm{ha}^{-1} \mathrm{fb}$ PoE application of pyrithiobac sodium $62.5 \mathrm{~g}$ $\mathrm{ha}^{-1}+$ quizalofop-p-ethyl $50 \mathrm{~g} \mathrm{ha}^{-1}$ at 20, 40, 60 DAS $\left(\mathrm{W}_{1}\right)$ and pendimethalin $1.0 \mathrm{~kg} \mathrm{ha}^{-1}(\mathrm{PE})$ $\mathrm{fb} \mathrm{HW}$ at 20 and 45 DAS $\left(\mathrm{W}_{3}\right)$, at all the stages except at 60 DAS where $\mathrm{W}_{1}$ in turn on par with $\mathrm{W}_{3}$ and significantly superior over unweeded control $\left(\mathrm{W}_{4}\right)$ treatment. But, at 30 DAS more main stem node number was observed with pendimethalin $1.0 \mathrm{~kg} \mathrm{ha}^{-1} \mathrm{fb}$ PoE application of pyrithiobac sodium $62.5 \mathrm{~g}$ $\mathrm{ha}^{-1}$ +quizalofop-p-ethyl $50 \mathrm{~g} \mathrm{ha}^{-1}$ at 20, 40, 60 DAS $\left(\mathrm{W}_{1}\right)$ and was on par with $\mathrm{W}_{2}$ and $\mathrm{W}_{3}$ treatments and were significantly superior over unweeded control $\left(\mathrm{W}_{4}\right)$ treatment.

In the present study, the increase in main stem node number in early PoE tank mix application of pyrithiobac sodium $62.5 \mathrm{~g} \mathrm{ha}^{-1}$ quizalofop-p-ethyl $50 \mathrm{~g} \mathrm{ha}^{-1}$ at 15 DAS fb directed spray of glyphosate ammonium salt $2.13 \mathrm{~kg} \mathrm{ha}^{-1}$ at $45 \mathrm{DAS}\left(\mathrm{W}_{2}\right)$ was might be due to increased plant height as evidenced from the Table 1.

\section{Interaction effect}

Interaction effect of plant densities and weed management practices did not show any significant influence on main stem node number of Bt cotton at all the crop growth stages.

\section{Monopodial branches (No. plant ${ }^{-1}$ )}

\section{Effect of plant densities}

Gradual increase in number of monopodial branches plant ${ }^{-1}$ was observed from 60 DAS to 120 DAS (Table 4). However, at 60 DAS plant density did not show any significant influence on number of monopodial branches plant $^{-1}$.

But, at 90 and 120 DAS more number of monopodia plant ${ }^{-1}$ was observed with plant density of 55,555 plants ha ${ }^{-1}\left(\mathrm{D}_{1}\right)$ and was on par with $1,11,111$ plants $\mathrm{ha}^{-1}\left(\mathrm{D}_{2}\right)$ normal planting and were significantly superior over 1,11,111 plants $\mathrm{ha}^{-1}\left(\mathrm{D}_{3}\right)$ paired row and $1,48,148$ plants $\mathrm{ha}^{-1}\left(\mathrm{D}_{4}\right)$ at 120 DAS, but 90 DAS $D_{2}$ and $D_{3}$ were on par with each other and were significantly superior over $1,48,148$ plants ha ${ }^{-1}\left(D_{4}\right)$ treatment.

\section{Effect of weed management practices}

Weed management practices showed significant influence on monopodia plant $^{-1}$ at all the stages. Significantly more monopodia plant $^{-1}$ were observed at 60 DAS with early PoE tank mix application of pyrithiobac sodium $62.5 \mathrm{~g} \mathrm{ha}^{-1}+$ quizalofop-p-ethyl $50 \mathrm{~g}$ $\mathrm{ha}^{-1}$ at 15 DAS fb directed spray of glyphosate ammonium salt $2.13 \mathrm{~kg} \mathrm{ha}^{-1}$ at $45 \mathrm{DAS}\left(\mathrm{W}_{2}\right)$ and was on par with pre emergence application of pendimethalin $1.0 \mathrm{~kg} \mathrm{ha}^{-1} \mathrm{fb}$ PoE tank mix application of pyrithiobac sodium $62.5 \mathrm{~g} \mathrm{ha}^{-1}+$ quizalofop-p-ethyl $50 \mathrm{~g}$ $\mathrm{ha}^{-1}$ at 20, 40, 60 DAS $\left(\mathrm{W}_{1}\right)$ and pendimethalin $1.0 \mathrm{~kg} \mathrm{ha}^{-1}(\mathrm{PE}) \mathrm{fb} \mathrm{HW}$ at 20 and 45 DAS $\left(\mathrm{W}_{3}\right)$. 
Table.1 Plant height of Bt cotton under varied plant densities and Weed management practices kharif, 2015

\begin{tabular}{|c|c|c|c|c|c|}
\hline \multirow{2}{*}{$\begin{array}{l}\text { Treatment } \\
\text { Plant densities (D) }\end{array}$} & \multicolumn{5}{|c|}{ Plant height $(\mathrm{cm})$} \\
\hline & $\begin{array}{c}\text { 30 } \\
\text { DAS }\end{array}$ & $\begin{array}{c}60 \\
\text { DAS }\end{array}$ & $\begin{array}{c}90 \\
\text { DAS }\end{array}$ & $\begin{array}{c}120 \\
\text { DAS }\end{array}$ & $\begin{array}{c}\text { At } \\
\text { harvest }\end{array}$ \\
\hline $\mathrm{D}_{1}-60 \mathrm{~cm} \times 30 \mathrm{~cm}(55,555)$ & 18 & 51 & 92 & 112 & 125 \\
\hline $\mathrm{D}_{2}-60 \mathrm{~cm} \times 15 \mathrm{~cm}(1,11,111)$ & 18 & 53 & 80 & 102 & 116 \\
\hline $\mathrm{D}_{3}-60 \mathrm{~cm} \times 15 \mathrm{~cm}(1,11,111$ Paired row- $45 \mathrm{~cm} \times 75 \mathrm{~cm})$ & 18 & 48 & 84 & 105 & 124 \\
\hline $\mathrm{D}_{4}-45 \mathrm{~cm} \times 15 \mathrm{~cm}(1,48,148)$ & 18 & 44 & 87 & 110 & 122 \\
\hline S. Em \pm & 0.4 & 1.0 & 2.0 & 2.6 & 3.2 \\
\hline $\mathrm{CD}(\mathrm{P}=0.05)$ & NS & 3.02 & 5.94 & NS & NS \\
\hline Weed Management Practices (W) & & & & & \\
\hline $\begin{array}{l}\mathrm{W}_{1} \text {-Pendimethalin } 30 \% \text { EC } 1.0 \mathrm{~kg} \mathrm{ha}^{-1} \text { as PE fb PoE pyrithiobac } \\
\text { sodium } 62.5 \mathrm{~g} \mathrm{ha}^{-1}+\text { quizalofop-p-ethyl } 5 \% \text { EC } 50 \mathrm{~g} \mathrm{ha}^{-1} \text { at 20, 40, } 60 \\
\text { DAS }\end{array}$ & 20 & 53 & 89 & 109 & 125 \\
\hline $\begin{array}{l}\mathrm{W}_{2} \text {-Pyrithiobac sodium } 10 \% \text { EC } 62.5 \mathrm{~g} \mathrm{ha}^{-1}+\text { quizalofop-p-ethyl } 50 \mathrm{~g} \\
\text { ha }^{-1} \text { at } 15 \text { DAS as early PoE fb glyphosate } 71 \% \text { SG } 2.13 \mathrm{~kg} \mathrm{ha}^{-1} \text { at } 45 \\
\text { DAS }\end{array}$ & 17 & 58 & 94 & 124 & 130 \\
\hline $\mathrm{W}_{3}$-Pendimethalin $1.0 \mathrm{~kg} \mathrm{ha}^{-1}$ as PE fb HW at 20 and 45 DAS & 18 & 52 & 94 & 109 & 129 \\
\hline $\mathrm{W}_{4}$-Unweeded control & 17 & 32 & 67 & 86 & 104 \\
\hline S. Em \pm & 0.4 & 1.0 & 2.0 & 2.6 & 3.2 \\
\hline $\mathrm{CD}(\mathrm{P}=0.05)$ & 1.2 & 3.0 & 5.6 & 7.7 & 9.3 \\
\hline Interaction (D X W) & & & & & \\
\hline S. Em \pm & 0.8 & 2.0 & 4.1 & 5.3 & 6.4 \\
\hline $\mathrm{CD}(\mathrm{P}=0.05)$ & NS & NS & NS & NS & NS \\
\hline
\end{tabular}

Table.2 Crop drymatter of Bt cotton under varied plant densities and Weed management practices kharif, 2015

\begin{tabular}{|c|c|c|c|c|c|}
\hline \multirow{2}{*}{$\begin{array}{l}\text { Treatment } \\
\text { Plant densities (D) }\end{array}$} & \multicolumn{5}{|c|}{ Crop Drymatter $\left(\right.$ g plant $\left.^{-1}\right)$} \\
\hline & $\begin{array}{c}\text { 30 } \\
\text { DAS }\end{array}$ & $\begin{array}{c}\text { 60 } \\
\text { DAS }\end{array}$ & $\begin{array}{c}90 \\
\text { DAS }\end{array}$ & $\begin{array}{l}\text { 120 } \\
\text { DAS }\end{array}$ & $\begin{array}{c}\text { At } \\
\text { harvest }\end{array}$ \\
\hline $\mathrm{D}_{1}-60 \mathrm{~cm} \times 30 \mathrm{~cm}(55,555)$ & 3.45 & 27.54 & 69.20 & 76.99 & 106.50 \\
\hline $\mathrm{D}_{2}-60 \mathrm{~cm} \times 15 \mathrm{~cm}(1,11,111)$ & 4.44 & 27.10 & 58.33 & 73.99 & 96.21 \\
\hline $\mathrm{D}_{3}-60 \mathrm{~cm} \times 15 \mathrm{~cm}(1,11,111$ Paired row- $45 \mathrm{~cm} \times 75 \mathrm{~cm})$ & 4.33 & 20.00 & 50.01 & 64.34 & 86.08 \\
\hline $\mathrm{D}_{4}-45 \mathrm{~cm} \times 15 \mathrm{~cm}(1,48,148)$ & 4.25 & 19.3 & 33.22 & 54.18 & 84.67 \\
\hline S. Em \pm & 0.47 & 2.52 & 4.97 & 3.34 & 3.58 \\
\hline $\mathrm{CD}(\mathrm{P}=0.05)$ & NS & 7.32 & 14.43 & 9.69 & 10.41 \\
\hline \multicolumn{6}{|l|}{ Weed Management Practices (W) } \\
\hline $\begin{array}{l}\mathrm{W}_{1} \text {-Pendimethalin } 30 \% \text { EC } 1.0 \mathrm{~kg} \mathrm{ha}^{-1} \text { as PE fb PoE pyrithiobac } \\
\text { sodium } 62.5 \mathrm{~g} \mathrm{ha}^{-1}+\text { quizalofop-p-ethyl } 5 \% \text { EC } 50 \mathrm{~g} \mathrm{ha}^{-1} \text { at } 20,40 \text {, } \\
60 \text { DAS }\end{array}$ & 3.88 & 29.79 & 69.27 & 82.29 & 114.79 \\
\hline $\begin{array}{l}\mathrm{W}_{2} \text {-Pyrithiobac sodium } 10 \% \text { EC } 62.5 \mathrm{~g} \mathrm{ha}^{-1}+\text { quizalofop-p-ethyl } \\
50 \mathrm{~g} \text { ha }{ }^{-1} \text { at } 15 \text { DAS as early PoE fb glyphosate } 71 \% \text { SG } 2.13 \mathrm{~kg} \\
\mathrm{ha}^{-1} \text { at } 45 \text { DAS }\end{array}$ & 5.08 & 25.50 & 51.25 & 70.97 & 99.167 \\
\hline $\mathrm{W}_{3}$-Pendimethalin $1.0 \mathrm{~kg} \mathrm{ha}^{-1}$ as PE fb HW at 20 and 45 DAS & 3.83 & 28.46 & 58.33 & 75.81 & 105.75 \\
\hline $\mathrm{W}_{4}$-Unweeded control & 3.70 & 10.19 & 31.91 & 40.43 & 53.75 \\
\hline S. Em \pm & 0.47 & 2.52 & 4.97 & 3.34 & 3.58 \\
\hline $\mathrm{CD}(\mathrm{P}=0.05)$ & NS & 7.32 & 14.43 & 9.69 & 10.41 \\
\hline \multicolumn{6}{|l|}{ Interaction (D X W) } \\
\hline S. Em \pm & 0.95 & 5.04 & 9.95 & 6.67 & 7.17 \\
\hline $\mathrm{CD}(\mathrm{P}=0.05)$ & NS & NS & NS & NS & NS \\
\hline
\end{tabular}


Table.3 Main stem nodes of Bt cotton under varied plant densities and Weed management practices kharif, 2015

\begin{tabular}{|c|c|c|c|c|c|}
\hline Treatment & \multicolumn{5}{|c|}{ Nodes (No. plant ${ }^{-1}$ ) } \\
\hline Plant densities (D) & 30 DAS & 60 DAS & 90 DAS & 120 DAS & At harvest \\
\hline $\mathrm{D}_{1}-60 \mathrm{~cm} \times 30 \mathrm{~cm}(55,555)$ & 6.72 & 14.12 & 20.22 & 24.35 & 26.67 \\
\hline $\mathrm{D}_{2}-60 \mathrm{~cm} \times 15 \mathrm{~cm}(1,11,111)$ & 6.37 & 14.23 & 17.15 & 23.08 & 25.17 \\
\hline $\begin{array}{l}\mathrm{D}_{3}-60 \mathrm{~cm} \times 15 \mathrm{~cm}(1,11,111 \text { Paired row- } 45 \\
\mathrm{cm} \times 75 \mathrm{~cm})\end{array}$ & 6.22 & 13.78 & 18.30 & 23.27 & 25.27 \\
\hline $\mathrm{D}_{4^{-}} 45 \mathrm{~cm} \times 15 \mathrm{~cm}(1,48,148)$ & 5.90 & 13.70 & 18.05 & 24.15 & 26.13 \\
\hline S. Em \pm & 0.15 & 0.13 & 0.30 & 0.81 & 0.68 \\
\hline $\mathrm{CD}(\mathrm{P}=0.05)$ & 0.46 & 0.40 & 0.89 & NS & NS \\
\hline Weed Management Practices (W) & & & & & \\
\hline $\begin{array}{l}\mathrm{W}_{1} \text {-Pendimethalin } 30 \% \text { EC } 1.0 \mathrm{~kg} \mathrm{ha}^{-1} \text { as } \\
\text { PE fb PoE pyrithiobac sodium } 62.5 \mathrm{~g} \mathrm{ha}^{-} \\
\text {1+quizalofop-p-ethyl } 5 \% \text { EC } 50 \mathrm{~g} \mathrm{ha}^{-1} \text { at } \\
\text { 20, 40,60 DAS }\end{array}$ & 6.63 & 15.05 & 19.73 & 25.20 & 27.22 \\
\hline $\begin{array}{l}\mathrm{W}_{2} \text {-Pyrithiobac sodium } 10 \% \text { EC } 62.5 \mathrm{~g} \text { ha- } \\
1^{-}+\text {quizalofop-p-ethyl } 50 \mathrm{~g} \mathrm{ha}^{-1} \text { at } 15 \text { DAS as } \\
\text { early PoE fb glyphosate } 71 \% \text { SG } 2.13 \mathrm{~kg} \\
\mathrm{ha}^{-1} \text { at } 45 \text { DAS }\end{array}$ & 6.58 & 15.38 & 20.20 & 26.43 & 28.47 \\
\hline $\begin{array}{l}\mathrm{W}_{3} \text {-Pendimethalin } 1.0 \mathrm{~kg} \mathrm{ha}^{-1} \text { as PE fb HW } \\
\text { at } 20 \text { and } 45 \text { DAS }\end{array}$ & 6.25 & 14.95 & 19.90 & 24.67 & 26.52 \\
\hline $\mathrm{W}_{4}$-Unweeded control & 5.73 & 9.95 & 13.88 & 18.55 & 21.03 \\
\hline S. Em \pm & 0.15 & 0.13 & 0.30 & 0.81 & 0.68 \\
\hline $\mathrm{CD}(\mathrm{P}=0.05)$ & 0.46 & 0.40 & 0.89 & 2.36 & 1.99 \\
\hline Interaction (D X W) & & & & & \\
\hline S. Em \pm & 0.31 & 0.27 & 0.61 & 1.631 & 1.376 \\
\hline $\mathrm{CD}(\mathrm{P}=0.05)$ & NS & NS & NS & $\mathrm{NS}$ & NS \\
\hline
\end{tabular}

Table.4 Monopodial branches in Bt cotton under varied plant densities and Weed management practices kharif, 2015

\begin{tabular}{|c|c|c|c|}
\hline \multirow{2}{*}{$\begin{array}{l}\text { Treatment } \\
\text { Plant densities (D) } \\
\end{array}$} & \multicolumn{3}{|c|}{ Monopodial branches (No. plant $^{-1}$ ) } \\
\hline & 60 DAS & 90 DAS & 120 DAS \\
\hline $\mathrm{D}_{1}-60 \mathrm{~cm} \times 30 \mathrm{~cm}(55,555)$ & 0.92 & 1.87 & 2.43 \\
\hline $\mathrm{D}_{2}-60 \mathrm{~cm} \times 15 \mathrm{~cm}(1,11,111)$ & 0.88 & 1.83 & 2.35 \\
\hline $\mathrm{D}_{3}-60 \mathrm{~cm} \times 15 \mathrm{~cm}(1,11,111$ Paired row- $45 \mathrm{~cm} \times 75 \mathrm{~cm})$ & 0.83 & 1.62 & 2.12 \\
\hline $\mathrm{D}_{4}-45 \mathrm{~cm} \times 15 \mathrm{~cm}(1,48,148)$ & 0.82 & 1.45 & 1.83 \\
\hline S. Em \pm & 0.04 & 0.06 & 0.05 \\
\hline $\mathrm{CD}(\mathrm{P}=0.05)$ & NS & 0.19 & 0.16 \\
\hline \multicolumn{4}{|l|}{ Weed Management Practices (W) } \\
\hline $\begin{array}{l}\mathrm{W}_{1} \text {-Pendimethalin } 30 \% \text { EC } 1.0 \mathrm{~kg} \mathrm{ha}^{-1} \text { as PE fb PoE pyrithiobac sodium } \\
62.5 \mathrm{~g} \mathrm{ha}^{-1}+\text { quizalofop-p-ethyl } 5 \% \mathrm{EC}^{2} \mathrm{~g} \mathrm{ha}^{-1} \text { at } 20,40,60 \mathrm{DAS}\end{array}$ & 1.15 & 2.03 & 2.50 \\
\hline $\begin{array}{l}\mathrm{W}_{2} \text {-Pyrithiobac sodium } 10 \% \text { EC } 62.5 \mathrm{~g} \mathrm{ha}^{-1}+\text { quizalofop-p-ethyl } 50 \mathrm{~g} \mathrm{ha} \\
{ }^{-} \text {at } 15 \text { DAS as early PoE fb glyphosate } 71 \% \text { SG } 2.13 \mathrm{~kg} \mathrm{ha}^{-1} \text { at } 45 \text { DAS }\end{array}$ & 1.17 & 1.87 & 2.37 \\
\hline $\mathrm{W}_{3}$-Pendimethalin $1.0 \mathrm{~kg} \mathrm{ha}^{-1}$ as PE fb HW at 20 and 45 DAS & 1.13 & 1.87 & 2.38 \\
\hline $\mathrm{W}_{4}$-Unweeded control & 0.00 & 1.00 & 1.48 \\
\hline S. Em \pm & 0.04 & 0.06 & 0.05 \\
\hline $\mathrm{CD}(\mathrm{P}=0.05)$ & 0.11 & 0.19 & 0.16 \\
\hline \multicolumn{4}{|l|}{ Interaction (D X W) } \\
\hline S. Em & 0.08 & 0.13 & 0.11 \\
\hline $\mathrm{CD}(\mathrm{P}=0.05)$ & NS & NS & 0.33 \\
\hline
\end{tabular}


Table.4a Interaction effect of plant densities and weed management practices on monopodial Branches in Bt cotton kharif, 2015

\begin{tabular}{|l|c|c|c|c|c|}
\hline \multirow{2}{*}{ Plant densities } & \multicolumn{5}{|c|}{ Weed management practices } \\
\cline { 2 - 6 } & $\mathrm{W}_{1}$ & $\mathrm{~W}_{2}$ & $\mathrm{~W}_{3}$ & $\mathrm{~W}_{4}$ & Mean \\
\hline $\mathrm{D}_{1}$ & 2.87 & 2.73 & 2.60 & 1.53 & 2.43 \\
\hline $\mathrm{D}_{2}$ & 2.60 & 2.67 & 2.67 & 1.47 & 2.35 \\
\hline $\mathrm{D}_{3}$ & 2.33 & 2.27 & 2.47 & 1.40 & 2.12 \\
\hline $\mathrm{D}_{4}$ & 2.20 & 1.80 & 1.80 & 1.53 & 1.83 \\
\hline Mean & 2.50 & 2.37 & 2.38 & 1.48 & \\
\hline $\mathrm{S} . \mathrm{Em} \pm$ & 0.11 & & & \\
\hline $\mathrm{CD}(\mathrm{P}=0.05)$ & 0.33 &
\end{tabular}

Table.5 Height to node ratio in Bt cotton under varied plant densities and Weed management practices kharif, 2015

\begin{tabular}{|c|c|c|c|c|c|}
\hline Treatment & \multicolumn{5}{|c|}{ Height to node ratio } \\
\hline Plant densities (D) & $\begin{array}{c}\text { 30 } \\
\text { DAS }\end{array}$ & $\begin{array}{c}\text { 60 } \\
\text { DAS }\end{array}$ & $\begin{array}{c}90 \\
\text { DAS }\end{array}$ & $\begin{array}{c}120 \\
\text { DAS }\end{array}$ & $\begin{array}{c}\text { At } \\
\text { harvest }\end{array}$ \\
\hline $\mathrm{D}_{1}-60 \mathrm{~cm} \times 30 \mathrm{~cm}(55,555)$ & 2.64 & 3.56 & 4.55 & 4.60 & 4.71 \\
\hline $\mathrm{D}_{2}-60 \mathrm{~cm} \times 15 \mathrm{~cm}(1,11,111)$ & 2.89 & 3.71 & 4.71 & 4.47 & 4.64 \\
\hline $\begin{array}{l}D_{3}-60 \mathrm{~cm} \times 15 \mathrm{~cm}(1,11,111 \text { Paired row- } 45 \mathrm{~cm} \times 75 \\
\mathrm{cm})\end{array}$ & 2.91 & 3.41 & 4.60 & 4.55 & 4.97 \\
\hline $\mathrm{D}_{4}-45 \mathrm{~cm} \times 15 \mathrm{~cm}(1,48,148)$ & 3.06 & 3.22 & 4.88 & 4.64 & 4.74 \\
\hline S. Em \pm & 0.06 & 0.05 & 0.11 & 0.14 & 0.16 \\
\hline $\mathrm{CD}(\mathrm{P}=0.05)$ & 0.18 & 0.16 & NS & NS & NS \\
\hline \multicolumn{6}{|l|}{ Weed Management Practices (W) } \\
\hline $\begin{array}{l}\mathrm{W}_{1} \text {-Pendimethalin } 30 \% \mathrm{EC} 1.0 \mathrm{~kg} \mathrm{ha}^{-1} \text { as PE fb PoE } \\
\text { pyrithiobac sodium } 62.5 \mathrm{~g} \mathrm{ha}^{-1}+\text { quizalofop-p-ethyl } \\
5 \% \text { EC } 50 \mathrm{~g} \mathrm{ha}^{-1} \text { at } 20,40,60 \mathrm{DAS}\end{array}$ & 3.02 & 3.51 & 4.54 & 4.34 & 4.64 \\
\hline $\begin{array}{l}\mathrm{W}_{2} \text {-Pyrithiobac sodium } 10 \% \text { EC } 62.5 \mathrm{~g} \mathrm{ha}^{-} \\
{ }^{+} \text {quizalofop-p-ethyl } 50 \mathrm{~g} \mathrm{ha}^{-1} \text { at } 15 \text { DAS as early PoE } \\
\text { fb glyphosate } 71 \% \mathrm{SG} 2.13 \mathrm{~kg} \mathrm{ha}^{-1} \text { at } 45 \text { DAS }\end{array}$ & 2.65 & 3.79 & 4.66 & 4.68 & 4.56 \\
\hline $\begin{array}{l}\mathrm{W}_{3} \text {-Pendimethalin } 1.0 \mathrm{~kg} \mathrm{ha}^{-1} \text { as PE fb HW at } 20 \text { and } \\
45 \text { DAS }\end{array}$ & 2.84 & 3.50 & 4.72 & 4.55 & 4.90 \\
\hline $\mathrm{W}_{4}$-Unweeded control & 3.01 & 3.10 & 4.83 & 4.68 & 4.95 \\
\hline S. Em \pm & 0.06 & 0.05 & 0.11 & 0.14 & 0.16 \\
\hline $\mathrm{CD}(\mathrm{P}=0.05)$ & 0.18 & 0.16 & NS & NS & NS \\
\hline \multicolumn{6}{|l|}{ Interaction (D X W) } \\
\hline S. Em \pm & 0.13 & 0.11 & 0.23 & 0.29 & 0.32 \\
\hline $\mathrm{CD}(\mathrm{P}=0.05)$ & $\mathrm{NS}$ & 0.32 & NS & $\mathrm{NS}$ & $\mathrm{NS}$ \\
\hline
\end{tabular}


Table.6 Chlorophyll content (SPAD reading) in Bt cotton under varied plant densities and Weed management practices kharif, 2015

\begin{tabular}{|c|c|c|c|c|}
\hline \multirow{2}{*}{$\begin{array}{l}\text { Treatment } \\
\text { Plant densities (D) }\end{array}$} & \multicolumn{4}{|c|}{ SPAD reading } \\
\hline & $\begin{array}{c}\text { 60 } \\
\text { DAS }\end{array}$ & $\begin{array}{c}90 \\
\text { DAS }\end{array}$ & $\begin{array}{l}\text { 120 } \\
\text { DAS }\end{array}$ & $\begin{array}{c}\text { At } \\
\text { harvest }\end{array}$ \\
\hline $\mathrm{D}_{1}-60 \mathrm{~cm} \times 30 \mathrm{~cm}(55,555)$ & 32.61 & 32.60 & 38.24 & 41.43 \\
\hline $\mathrm{D}_{2}-60 \mathrm{~cm} \times 15 \mathrm{~cm}(1,11,111)$ & 32.10 & 32.10 & 35.03 & 40.66 \\
\hline $\mathrm{D}_{3}-60 \mathrm{~cm} \times 15 \mathrm{~cm}(1,11,111$ Paired row- $45 \mathrm{~cm} \times 75 \mathrm{~cm})$ & 32.04 & 32.04 & 35.94 & 42.43 \\
\hline $\mathrm{D}_{4}-45 \mathrm{~cm} \times 15 \mathrm{~cm}(1,48,148)$ & 32.42 & 32.42 & 35.77 & 41.54 \\
\hline S. Em \pm & 0.98 & 1.02 & 0.44 & 0.76 \\
\hline $\mathrm{CD}(\mathrm{P}=0.05)$ & NS & NS & 1.27 & NS \\
\hline \multicolumn{5}{|l|}{ Weed management practices $(\mathrm{W})$} \\
\hline $\begin{array}{l}\mathrm{W}_{1} \text {-Pendimethalin } 30 \% \text { EC } 1.0 \mathrm{~kg} \mathrm{ha}^{-1} \text { as PE fb PoE pyrithiobac sodium } \\
62.5 \mathrm{~g} \mathrm{ha}^{-1}+\text { quizalofop-p-ethyl } 5 \% \mathrm{EC}^{2} \mathrm{~g} \mathrm{ha}^{-1} \text { at } 20,40,60 \mathrm{DAS}\end{array}$ & 34.12 & 35.02 & 36.99 & 42.20 \\
\hline $\begin{array}{l}\mathrm{W}_{2} \text {-Pyrithiobac sodium } 10 \% \text { EC } 62.5 \mathrm{~g} \mathrm{ha}^{-1}+\text { quizalofop-p-ethyl } 50 \mathrm{~g} \text { ha- } \\
{ }^{1} \text { at } 15 \text { DAS as early PoE fb glyphosate } 71 \% \text { SG } 2.13 \mathrm{~kg} \mathrm{ha}^{-1} \text { at } 45 \text { DAS }\end{array}$ & 32.24 & 32.24 & 38.20 & 42.72 \\
\hline $\mathrm{W}_{3}$-Pendimethalin $1.0 \mathrm{~kg} \mathrm{ha}^{-1}$ as PE fb HW at 20 and 45 DAS & 33.88 & 34.4 & 37.64 & 41.77 \\
\hline $\mathrm{W}_{4}$-Unweeded control & 28.93 & 33.58 & 32.82 & 39.38 \\
\hline S. Em \pm & 0.98 & 1.02 & 0.44 & 0.76 \\
\hline $\mathrm{CD}(\mathrm{P}=0.05)$ & 2.87 & 2.97 & 1.27 & 2.23 \\
\hline \multicolumn{5}{|l|}{ Interaction (D X W) } \\
\hline S. Em \pm & 1.97 & 2.04 & 0.894 & 1.53 \\
\hline $\mathrm{CD}(\mathrm{P}=0.05)$ & NS & NS & NS & NS \\
\hline
\end{tabular}

Table.7 Phenology of Bt cotton under varied plant densities and Weed management practices kharif, 2015

\begin{tabular}{|c|c|c|c|c|}
\hline Treatment & $\begin{array}{c}\text { Days to } \\
\text { first } \\
\text { square } \\
\text { formatoin }\end{array}$ & $\begin{array}{c}\text { Days } \\
\text { to } \\
\text { first } \\
\text { flower } \\
\text { open }\end{array}$ & $\begin{array}{l}\text { Days } \\
\text { to } \\
\text { first } \\
\text { boll } \\
\text { open }\end{array}$ & $\begin{array}{c}\text { Earli- } \\
\text { ness } \\
\text { index }\end{array}$ \\
\hline \multicolumn{5}{|l|}{ Plant densities (D) } \\
\hline $\mathrm{D}_{1}-60 \mathrm{~cm} \times 30 \mathrm{~cm}(55,555)$ & 35 & 52 & 106 & 46.31 \\
\hline $\mathrm{D}_{2}-60 \mathrm{~cm} \times 15 \mathrm{~cm}(1,11,111)$ & 37 & 52 & 106 & 56.94 \\
\hline $\mathrm{D}_{3}-60 \mathrm{~cm} \times 15 \mathrm{~cm}(1,11,111$ Paired row- $45 \mathrm{~cm} \times 75 \mathrm{~cm})$ & 37 & 51 & 108 & 51.39 \\
\hline $\mathrm{D}_{4}-45 \mathrm{~cm} \times 15 \mathrm{~cm}(1,48,148)$ & 38 & 51 & 113 & 39.65 \\
\hline S. Em \pm & 0.17 & 0.4 & 0.4 & 4.26 \\
\hline $\mathrm{CD}(\mathrm{P}=0.05)$ & 0.5 & NS & 1.1 & 12.38 \\
\hline \multicolumn{5}{|l|}{ Weed Management Practices $(\mathrm{W})$} \\
\hline $\begin{array}{l}\mathrm{W}_{1} \text {-Pendimethalin } 30 \% \text { EC } 1.0 \mathrm{~kg} \mathrm{ha}^{-1} \text { as PE fb PoE pyrithiobac sodium } 62.5 \\
\mathrm{~g} \mathrm{ha}^{-1}+\text { quizalofop-p-ethyl 5\% EC } 50 \mathrm{~g} \mathrm{ha}^{-1} \text { at 20, 40, 60 DAS }\end{array}$ & 36 & 51 & 106 & 63.09 \\
\hline $\begin{array}{l}\mathrm{W}_{2} \text {-Pyrithiobac sodium } 10 \% \text { EC } 62.5 \mathrm{~g} \mathrm{ha}^{-1}+\text { quizalofop-p-ethyl } 50 \mathrm{~g} \mathrm{ha}^{-1} \text { at } 15 \\
\text { DAS as early PoE fb glyphosate } 71 \% \mathrm{SG} 2.13 \mathrm{~kg} \mathrm{~h}^{-1} \text { at } 45 \mathrm{DAS}\end{array}$ & 36 & 52 & 106 & 53.73 \\
\hline $\mathrm{W}_{3}$-Pendimethalin $1.0 \mathrm{~kg} \mathrm{ha}^{-1}$ as PE fb HW at 20 and 45 DAS & 36 & 51 & 105 & 52.51 \\
\hline $\mathrm{W}_{4}$-Unweeded control & 38 & 53 & 116 & 24.95 \\
\hline S. Em \pm & 0.17 & 0.4 & 0.4 & 4.26 \\
\hline $\mathrm{CD}(\mathrm{P}=0.05)$ & 0.5 & 1.0 & 1.1 & 12.38 \\
\hline \multicolumn{5}{|l|}{ Interaction (D X W) } \\
\hline S. Em \pm & 0.34 & 0.73 & 0.76 & 8.53 \\
\hline $\mathrm{CD}(\mathrm{P}=0.05)$ & 1.0 & NS & NS & 24.76 \\
\hline
\end{tabular}


Table.7a Interaction effect of plant densities and weed management practices on earliness index in Bt cotton kharif-2015

\begin{tabular}{|l|c|c|c|c|c|}
\hline \multicolumn{5}{|c|}{ Earliness index } \\
\hline $\mathrm{D} / \mathrm{W}$ & $\mathrm{W}_{1}$ & $\mathrm{~W}_{2}$ & $\mathrm{~W}_{3}$ & $\mathrm{~W}_{4}$ & Mean \\
\hline $\mathrm{D}_{1}$ & 52.94 & 40.98 & 48.31 & 43.03 & 46.31 \\
\hline $\mathrm{D}_{2}$ & 60.93 & 75.09 & 64.80 & 26.96 & 56.95 \\
\hline $\mathrm{D}_{3}$ & 88.01 & 59.78 & 48.58 & 9.19 & 51.39 \\
\hline $\mathrm{D}_{4}$ & 50.51 & 39.06 & 48.38 & 20.65 & 39.65 \\
\hline Mean & 63.10 & 53.73 & 52.52 & 24.96 & \\
\hline $\mathrm{S} . \mathrm{Em} \pm$ & 8.53 & \multicolumn{5}{l}{} \\
\hline $\mathrm{CD}(\mathrm{P}=0.05)$ & 24.76 &
\end{tabular}

However, at 90 DAS and 120 DAS significantly more monopodia plant $^{-1}$ was noticed with pre emergence application of pendimethalin $1.0 \mathrm{~kg} \mathrm{ha}^{-1} \mathrm{fb}$ PoE tank mix application of pyrithiobac sodium $62.5 \mathrm{~g} \mathrm{ha}^{-}$ ${ }^{1}$ +quizalofop-p-ethyl $50 \mathrm{~g} \mathrm{ha}^{-1}$ at 20, 40, 60 DAS $\left(\mathrm{W}_{1}\right)$ and was on par with $1.0 \mathrm{~kg} \mathrm{ha}^{-1}$ (PE) fb HW at 20 and 45 DAS $\left(\mathrm{W}_{3}\right)$ and early PoE tank mix application of pyrithiobac sodium $62.5 \mathrm{~g} \mathrm{ha}^{-1}+$ quizalofop-p-ethyl $50 \mathrm{~g}$ $\mathrm{ha}^{-1}$ at $15 \mathrm{DAS}$ fb directed spray of glyphosate ammonium salt $2.13 \mathrm{~kg} \mathrm{ha}^{-1}$ at $45 \mathrm{DAS}\left(\mathrm{W}_{2}\right)$ treatments. Significantly the lower number of monopodia plant $^{-1}$ was recorded with unweeded control $\left(\mathrm{W}_{4}\right)$ treatment.

\section{Interaction effect}

Interaction effect of plant densities and weed management practices showed significant influence on number of monopodial branches plant $^{-1}$ of Bt cotton at 120 DAS stage only (Table 4a).

Plant density of 55,555 plants ha ${ }^{-1}\left(\mathrm{D}_{1}\right)$ and $1,11,111$ plants $\mathrm{ha}^{-1}\left(\mathrm{D}_{2}\right)$ normal planting along with application of either pre emergence application of pendimethalin 1.0 $\mathrm{kg} \mathrm{ha}{ }^{-1} \mathrm{fb}$ PoE tank mix application of pyrithiobac sodium $62.5 \mathrm{~g} \mathrm{ha}^{-1}+$ quizalofop-pethyl $50 \mathrm{~g} \mathrm{ha}^{-1}$ at 20, 40, 60 DAS $\left(\mathrm{W}_{1}\right)$ or early PoE tank mix application of pyrithiobac sodium $62.5 \mathrm{~g} \mathrm{ha}^{-1}+$ quizalofop-p-ethyl $50 \mathrm{~g}$ $\mathrm{ha}^{-1}$ at 15 DAS fb directed spray of glyphosate ammonium salt $2.13 \mathrm{~kg} \mathrm{ha}^{-1}$ at $45 \mathrm{DAS}\left(\mathrm{W}_{2}\right)$ or pendimethalin $1.0 \mathrm{~kg} \mathrm{ha}^{-1}(\mathrm{PE}) \mathrm{fb} \mathrm{HW}$ at 20 and 45 DAS $\left(\mathrm{W}_{3}\right)$ showed significantly more and on par number of monopodial branches plant ${ }^{-1}$.

These were followed by $1,11,111$ plants $\mathrm{ha}^{-1}$ $\left(D_{3}\right)$ paired row planting with all weed management practices. However, plant density of $1,48,148$ plants $\mathrm{ha}^{-1}\left(\mathrm{D}_{4}\right)$ showed only increased number with weed management practice, pre emergence application of pendimethalin $1.0 \mathrm{~kg} \mathrm{ha}^{-1} \mathrm{fb}$ PoE tank mix application of pyrithiobac sodium $62.5 \mathrm{~g} \mathrm{ha}^{-1}+$ quizalofop-p-ethyl $50 \mathrm{~g}$ $\mathrm{ha}^{-1}$ at 20, 40, 60 DAS $\left(\mathrm{W}_{1}\right)$. Significantly less number of monopodial branches plant ${ }^{-1}$ was recorded with unweeded control $\left(\mathrm{W}_{4}\right)$ at all levels of plant densities.

\section{Height to node ratio}

\section{Effect of plant densities}

Plant density did not show any significant influence on height to node ratio at 90, 120 DAS and at final harvest stage (Table 5). But, at 30 DAS significantly higher height to node ratio was observed with $1,48,148$ plants $\mathrm{ha}^{-1}$ $\left(D_{4}\right)$ and was on par with 1,11,111 plants ha ${ }^{-1}$ $\left(D_{3}\right)$ paired row planting and 1,11,111 plants ha $^{-1}\left(\mathrm{D}_{2}\right)$ normal planting, significantly lower height to node ratio was observed with 55,555 plants ha ${ }^{-1}\left(D_{1}\right)$. However, at 60 DAS more 
height to node ratio was noticed with $1,11,111$ plants ha ${ }^{-1}\left(D_{2}\right)$ normal planting and was on par with 55,555 plants ha ${ }^{-1}\left(D_{1}\right)$ and $1,11,111$ plants ha ${ }^{-1}\left(\mathrm{D}_{3}\right)$ paired row planting, in turn lower height to node ratio observed with $1,48,148$ plants $\mathrm{ha}^{-1}\left(\mathrm{D}_{4}\right)$ treatment. The increased height to node ratio in these treatments might be due to increased plant height at 60 DAS with low plant densities. But at later stages increased plant height and reduced node number increased the height to node ratio at non-significant level.

The enhanced height to node ratio at closer spacings might be due to higher inter plant competition at higher plant populations (Jonathan et al., 2006). The present results were similar to observations of Siebert and Stewart (2006).

\section{Effect of weed management practices}

Weed management practices did not show any significant influence on height to node ratio at 90, 120 and at final harvest, but at 30 DAS significantly more height to node ratio was recorded with pre emergence application of pendimethalin $1.0 \mathrm{~kg} \mathrm{ha}^{-1} \mathrm{fb}$ PoE tank mix application of pyrithiobac sodium $62.5 \mathrm{~g}$ ha

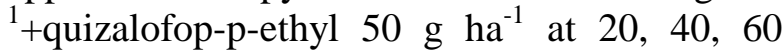
DAS $\left(\mathrm{W}_{1}\right)$ and was on par with unweeded control $\left(\mathrm{W}_{4}\right)$ and pendimethalin $1.0 \mathrm{~kg} \mathrm{ha}^{-1}(\mathrm{PE})$ $\mathrm{fb} \mathrm{HW}$ at 20 and 45 DAS $\left(\mathrm{W}_{3}\right)$ treatments and were significantly superior over early PoE tank mix application of pyrithiobac sodium $62.5 \mathrm{~g}$ $\mathrm{ha}^{-1}+$ quizalofop-p-ethyl $50 \mathrm{~g} \mathrm{ha}^{-1}$ at $15 \mathrm{DAS} \mathrm{fb}$ directed spray of glyphosate ammonium salt $2.13 \mathrm{~kg} \mathrm{ha}^{-1}$ at $45 \mathrm{DAS}\left(\mathrm{W}_{2}\right)$. But, at $60 \mathrm{DAS}$ significantly more height to node ratio was recorded with early PoE tank mix application of pyrithiobac sodium $62.5 \mathrm{~g} \mathrm{ha}^{-1}+$ quizalofop-pethyl $50 \mathrm{~g} \mathrm{ha}^{-1}$ at 15 DAS fb directed spray of glyphosate ammonium salt $2.13 \mathrm{~kg} \mathrm{ha}^{-1}$ at 45 DAS $\left(\mathrm{W}_{2}\right)$ and was significantly superior over rest of other treatments, this was followed by pre emergence application of pendimethalin 1.0 $\mathrm{kg} \mathrm{ha}{ }^{-1} \mathrm{fb}$ PoE tank mix application of pyrithiobac sodium $62.5 \mathrm{~g} \mathrm{ha}^{-1}+$ quizalofop-p- ethyl $50 \mathrm{~g} \mathrm{ha}^{-1}$ at 20, 40, 60 DAS $\left(\mathrm{W}_{1}\right)$ and pendimethalin $1.0 \mathrm{~kg} \mathrm{ha}^{-1}$ (PE) fb HW at 20 and 45 DAS $\left(\mathrm{W}_{3}\right)$. These were on par with each other and significantly superior over unweeded control $\left(\mathrm{W}_{4}\right)$ treatment.

\section{Interaction effect}

Interaction effect of plant densities and weed management practices did not show any significant influence on height to node ratio of Bt cotton at all the crop growth stages.

\section{SPAD value}

\section{Effect of plant densities}

Plant density did not show any variation in chlorophyll meter value at 60 DAS, 90 DAS and at final harvest stage (Table 6). But at 120 DAS siginicantly higher SPAD value was recorded with $55,555\left(\mathrm{D}_{1}\right)$ plants $\mathrm{ha}^{-1}$ and was followed by $1,11,111\left(D_{2}\right)$ plants $\mathrm{ha}^{-1}$ normal planting, 1,11,111 $\left(\mathrm{D}_{3}\right)$ plants $\mathrm{ha}^{-1}$ paired row and $1,48,148$ plants $\mathrm{ha}^{-1}\left(\mathrm{D}_{4}\right)$ and were on par with each other.

\section{Effect of weed management practices}

Weed management practices showed significant difference in SPAD values at different growth stages. At 60 and 90 DAS significantly more SPAD value or chlorophyll meter value recorded with pre emergence application of pendimethalin $1.0 \mathrm{~kg} \mathrm{ha}^{-1} \mathrm{fb}$ PoE application of pyrithiobac sodium $62.5 \mathrm{~g} \mathrm{ha}^{-1}+$ quizalofop-pethyl $50 \mathrm{~g} \mathrm{ha}^{-1}$ at 20, 40, 60 DAS (W W $_{1}$ ) and was on par with pendimethalin $1.0 \mathrm{~kg} \mathrm{ha}^{-1}$ (PE) fb $\mathrm{HW}$ at 20 and 45 DAS $\left(\mathrm{W}_{3}\right)$ and early PoE tank mix application of pyrithiobac sodium $62.5 \mathrm{~g}$ $\mathrm{ha}^{-1}+$ quizalofop-p-ethyl $50 \mathrm{~g} \mathrm{ha}^{-1}$ at 15 DAS fb directed spray of glyphosate ammonium salt $2.13 \mathrm{~kg} \mathrm{ha}^{-1}$ at $45 \mathrm{DAS}\left(\mathrm{W}_{2}\right)$. But, at $120 \mathrm{DAS}$ and final harvest significantly higher SPAD meter values noticed with early PoE tank mix application of pyrithiobac sodium $62.5 \mathrm{~g}$ ha ${ }^{1}$ +quizalofop-p-ethyl $50 \mathrm{~g} \mathrm{ha}^{-1}$ at $15 \mathrm{DAS} \mathrm{fb}$ directed spray of glyphosate ammonium salt 
$2.13 \mathrm{~kg} \mathrm{ha}^{-1}$ at $45 \mathrm{DAS}\left(\mathrm{W}_{2}\right)$ and was on par with pendimethalin $1.0 \mathrm{~kg} \mathrm{ha}^{-1}(\mathrm{PE}) \mathrm{fb} \mathrm{HW}$ at 20 and 45 DAS $\left(\mathrm{W}_{3}\right)$ and pre emergence application of pendimethalin $1.0 \mathrm{~kg} \mathrm{ha}^{-1} \mathrm{fb} \mathrm{PoE}$ application of pyrithiobac sodium $62.5 \mathrm{~g}^{-}$ ${ }^{1}$ +quizalofop-p-ethyl $50 \mathrm{~g} \mathrm{ha}^{-1}$ at $20,40,60$ DAS $\left(\mathrm{W}_{1}\right)$. The lowest spad values at all the growth stages were recorded with unweeded control $\left(\mathrm{W}_{4}\right)$ treatment. This might be due to presence of more weeds.

\section{Interaction effect}

Plant densities and weed management practices did not show any significant influence on SPAD reading value of Bt cotton at 60 DAS, 90 DAS and 120 DAS.

\section{Phenology}

\section{Days to first square initiation}

\section{Effect of plant densities}

Early square initiation was observed with 55,555 plants ha ${ }^{-1}\left(D_{1}\right)$, which was significantly superior over rest of the plant densities and was followed by plant density of 1,11,111 plants $\mathrm{ha}^{-1}$ $\left(\mathrm{D}_{2}\right)$ normal planting, in turn this was on par with $1,11,111$ plants $\mathrm{ha}^{-1}\left(\mathrm{D}_{3}\right)$ paired row planting. Significantly more number of days took to attain square initiation with $1,48,148$ plants ha ${ }^{-1}\left(\mathrm{D}_{4}\right)$ (Table 7$)$.

Number of days from planting to first floral bud initiation (squaring) were significantly decreased by narrow row spacing might be due to increased inter plant competition (Munir et al., 2015). These results substantiated the findings of Bednarz et al., (2000) and Mygdakos et al., (2004), who reported that the earliness (early squaring or flowering) increased when row spacing decreased.

\section{Effect of weed management practices}

Weed management practices showed significant influence on square initiation. Significantly lesser number of days taken to attain first square with pre emergence application of pendimethalin $1.0 \mathrm{~kg} \mathrm{ha}^{-1} \mathrm{fb}$ PoE tank mix application of pyrithiobac sodium $62.5 \mathrm{~g}$ ha ${ }^{1}$ +quizalofop-p-ethyl $50 \mathrm{~g} \mathrm{ha}^{-1}$ at 20, 40, 60 DAS $\left(\mathrm{W}_{1}\right)$ and was on par with pendimethalin $1.0 \mathrm{~kg} \mathrm{ha}^{-1}$ (PE) fb HW at 20 and 45 DAS $\left(\mathrm{W}_{3}\right)$ and early PoE tank mix application of pyrithiobac sodium $62.5 \mathrm{~g} \mathrm{ha}^{-1}+$ quizalofop-pethyl $50 \mathrm{~g} \mathrm{ha}^{-1}$ at 15 DAS fb directed spray glyphosate ammonium salt $2.13 \mathrm{~kg} \mathrm{ha}^{-1}$ at 45 DAS $\left(\mathrm{W}_{2}\right)$, however, unweeded control $\left(\mathrm{W}_{4}\right)$ treatment taken more number of days to attain first square. This was due to presence of weeds along the cotton crop might have resulted in delay in square initiation due to reduced availability of resources to cotton crop.

\section{Interaction effect}

Significantly early square initiation was observed with population density of 55,555 plants $\mathrm{ha}^{-1}\left(\mathrm{D}_{1}\right)$ along with pre emergence application pendimethalin $1.0 \mathrm{~kg} \mathrm{ha}^{-1} \mathrm{fb} \mathrm{PoE}$ application of pyrithiobac sodium $62.5 \mathrm{~g} \mathrm{ha}^{-}$ ${ }^{1}$ +quizalofop-p-ethyl $50 \mathrm{~g} \mathrm{ha}^{-1}$ at 20, 40, 60 DAS $\left(\mathrm{W}_{1}\right)$, early PoE application of pyrithiobac sodium $62.5 \mathrm{~g} \mathrm{ha}^{-1}+$ quizalofop-p-ethyl $50 \mathrm{~g} \mathrm{ha}^{-}$ ${ }^{1}$ at 15 DAS fb glyphosate ammonium salt 2.13 $\mathrm{kg} \mathrm{ha}^{-1}$ at 45 DAS $\left(\mathrm{W}_{2}\right)$ and pendimethalin 1.0 $\mathrm{kg} \mathrm{ha}^{-1}(\mathrm{PE}) \mathrm{fb} \mathrm{HW}$ at 20 and 45 DAS $\left(\mathrm{W}_{3}\right)$, and these were followed by population density of 1,11,111 plants $\mathrm{ha}^{-1}$ normal planting and paired row planting along with pre emergence application pendimethalin $1.0 \mathrm{~kg} \mathrm{ha}^{-1} \mathrm{fb} \mathrm{PoE}$ application of pyrithiobac sodium $62.5 \mathrm{~g} \mathrm{ha}^{-}$ ${ }^{1}$ +quizalofop-p-ethyl $50 \mathrm{~g} \mathrm{ha}^{-1}$ at 20, 40, 60 DAS $\left(\mathrm{W}_{1}\right)$, early PoE tank mix application of pyrithiobac sodium $62.5 \mathrm{~g} \mathrm{ha}^{-1}+$ quizalofop-pethyl $50 \mathrm{~g} \mathrm{ha}^{-1}$ at 15 DAS fb glyphosate ammonium salt $2.13 \mathrm{~kg} \mathrm{ha}^{-1}$ at $45 \mathrm{DAS}\left(\mathrm{W}_{2}\right)$ and pendimethalin $1.0 \mathrm{~kg} \mathrm{ha}^{-1}$ (PE) fb $\mathrm{HW}$ at 20 and 45 DAS $\left(\mathrm{W}_{3}\right)$. whereas plant density of 1,48,148 plants ha $^{-1}$ along with these weed management practices taken more number of days to attain the first square initiation. At all the plant densities unweeded control $\left(\mathrm{W}_{4}\right)$ has taken more number of days to get the first square initiation. 


\section{References}

Ali, H., Abid, S.S., Ahmad, S., Sarwar, N., Arooj, M., Mahmood, A and Shahzad, A.N. 2013. Impact of integrated weed management on flat-sown cotton (Gossypium hirsutum L.). The Journal of Animal and Plant Sciences. 23 (4): 11851192.

Anonymous, 2016. http://cotcorp.gov.in

Bednarz, C.W., Bridges, D.C and Brown, S.M. 2000. Analysis of cotton yield stability across population densities. Agronomy Journal. 92: 128-135.

Bhalerao, P.D., Patil, P.U., Ghatol, P.U and Gawande, P.P. 2010. Effect of spacing and fertilizer levels on seed cotton yield under rainfed condition. Indian Journal of Agriculture Research. 44 (10): 74-76.

Brar, S., R.J.S. Thindand L.S.Brar,Integrated weed control in upland cotton (Gossypiumhirsutum L.). Indian J. Weed Sci., 27(3\&4): 138-143.1995.

Devraj, Bhattoo, M.S., Duhan, B.S., Kumari, P and Jain, P.P. 2011. Effect of geometry and fertilizer levels on seed cotton yield and nutrient uptake of $\mathrm{Bt}$ cotton under irrigated conditions. Journal of Cotton Research and Deelopment. 25 (2):176180.

Dhillon, G.S., Chabra, K.L and Punia, S.S. 2006. Effect of crop geometry and integrated nutrient management on fibre quality and nutrient uptake by cotton crop. Journal of Cotton Research and Development. 20 (2): 221-223.

Hiremath, R., Gurappa, S.Y., Basavaraj, M.C, Ayyanna, D.S., Vidyavathi, G.Y., Rao, B and Koppolkar, G. 2013. Efficacy of chemical weed management in Bt cotton. Acta Biological Indica. 2 (2): 425-429.

Jain, S.C., 1982. Nutrient competition and weed control in cotton. Journal of the Indian society for cotton improvement. 7: 25-30.

Jalis, A., and Shah, M.L. 1982. Weed control in cotton resume. The Pak Cottons. 26 (3):141-148

Jonathan, D., Siebert and Alexander, M., Stewart. 2006. Influence of plant density on cotton response to mepiquat chloride application. Agronomy Journal. 98: 16341639.

Munir, M.K., Tahir, M., Saleem, M.F and Yaseen, M. 2015. Growth, yield and earliness response of cotton to row spacing and nitrogen management. The Journal of Animal \& Plant Sciences. 25 (3): 729-738.

Mygdakos, E., Avgoulas, C., Adamidis, K and Ekonomou, G. 2004. Cotton cultivation on narrow rows as an alternative culture method: Production and economics. Journal of Food, Agriculture and Environment. 2: 198-201.

Pawar, A.K., B.M. Patil., A.P. Karunakar and Satao, R.N.2000. Effect of pre and post emergence herbicides on weed control and yield of cotton. Pestology. 24: 13-17.

Siebert, J.D., and Stewart, A.M. 2006. Influence of plant density on cotton response to mepiquat chloride application. Agronomy Journal. 98: 1634-1639.

Venugopalan, M.V., Sankaranarayanan, K., Blaise, D., Nalayini, P., prahraj, C. S. and Gangaiah, B. 2009. Bt cotton (Gossypium sp.) in India and its agronomic requirements A review. Indian $J$. of Agronomy. 54 (4): 343-360.

Wang, G., Assimwe, R.K and Andrade, P. 2011. Growth and yield response to plant population of two cotton varieties with different growth habits. Arizona cotton report (161). Pp. 6-11.

\section{How to cite this article:}

Madavi, B., P. Leela Rani, G. Sreenivas and Surekha, K. 2017. Impact of High Density Planting and Weed Management Practices on Growth Parameters and Phenology of Bt Cotton. Int.J.Curr.Microbiol.App.Sci. 6(9): 2360-2372. doi: https://doi.org/10.20546/ijcmas.2017.609.289 